\title{
Satisfacción del turista en los eventos del FIAV para la declaratoria de "Loja, ciudad creativa de la música"
}

\section{Tourist satisfaction at the FIAV events for the declaration of "Loja, creative city of music"}

\begin{abstract}
Ana Carola Flores Mancheno. ${ }^{1}$, Claudia Patricia Maldonado Erazo. ${ }^{2}$, Nancy Patricia Tierra
\end{abstract} Tierra. ${ }^{3} \&$ Patricia de Lourdes Gallegos Murillo. ${ }^{4}$

\begin{abstract}
.
DOI: https://doi.org/10.33262/cienciadigital.v3i3.4..846

The "Creative Cities Network - UNESCO" initiative is established as a cluster of local development that groups cities characterized by their capabilities, whose objective is to work together to encourage cultural and creative industries. Within this framework, the city Loja has established as a goal of local development to join this network within the field of music. Cultural manifestations related to music have been articulated in the International Festival of Living Arts of Loja (FIAVL), an event that promotes spaces for the manifestation of several performing arts. This event has become a source of foreign exchange for the resident population, thanks to tourist flows. Based on this, the purpose of the study is to analyze the satisfaction of the tourist about the events of the FIAVL, through a correspondence analysis of the 2017 edition.
\end{abstract}

\footnotetext{
${ }^{1}$ Escuela Superior Politécnica de Chimborazo, Facultad de Recursos Naturales. Riobamba, Ecuador. acmancheno@espoch.edu.ec

${ }^{2}$ Escuela Superior Politécnica de Chimborazo, Facultad de Recursos Naturales. Riobamba, Ecuador. claudia.maldonado@espoch.edu.ec

${ }^{3}$ Escuela Superior Politécnica de Chimborazo, Facultad de Recursos Naturales. Riobamba, Ecuador. ntierra@espoch.edu.ec

${ }^{4}$ Escuela Superior Politécnica de Chimborazo, Facultad de Salud Pública. Riobamba, Ecuador. p_gallegos@espoch.edu.ec
} 
Keywords: Cultural heritage, tourism, correspondence analysis, music, case study.

\section{Resumen}

La iniciativa "Red de Ciudades Creativas - UNESCO", se establece como un clúster de desarrollo local que agrupa ciudades caracterizadas por sus capacidades, cuyo objetivo es trabajar de forma conjunta para incentivar las industrias culturales y creativas. En este marco, la ciudad Loja ha establecido como meta de desarrollo local anexarse a dicha red dentro del campo de la música. Las manifestaciones culturales relacionadas con la música se han articulado en el Festival Internacional de Artes Vivas de Loja (FIAVL), evento que promueve espacios para la manifestación de varias artes escénicas. Este evento se ha constituido como una fuente generadora de divisas para la población residente, gracias a los flujos turísticos. En función de ello, el propósito del estudio es analizar la satisfacción del turista sobre los eventos del FIAVL, a través de un análisis de correspondencia de la edición 2017.

Palabras claves: Patrimonio cultural, turismo, análisis de correspondencia, música, estudio de caso.

\section{Introducción.}

Ecuador, se integra por 24 provincias que se organizan en nueve zonas de planificación. Puntualmente, la zona 7 se compone de las provincias de El Oro, Loja y Zamora Chinchipe, la cual representa la franja sur del país, o también conocida como Zona de Integración Fronteriza (ZIF) con la República del Perú.

La ciudad de Loja destaca dentro de la zona de planificación, por establecerse históricamente a nivel nacional como "Cuna de artistas", gracias a la alta concentración de emblemáticos representantes de la literatura, música, pintura, entre otros, como fueron: Benjamín Carrión, Eduardo Kingman, Ángel Felicísimo Rojas, Pablo Palacio, Segundo Cueva Celi y Matilde Hidalgo de Procel; quienes han contribuido en la generación de la expresión "el que no toca la guitarra puede cantar una canción; el que no canta una canción puede escribir un verso; el que no escribe un verso se lee en un libro" (Oviedo 2012). 


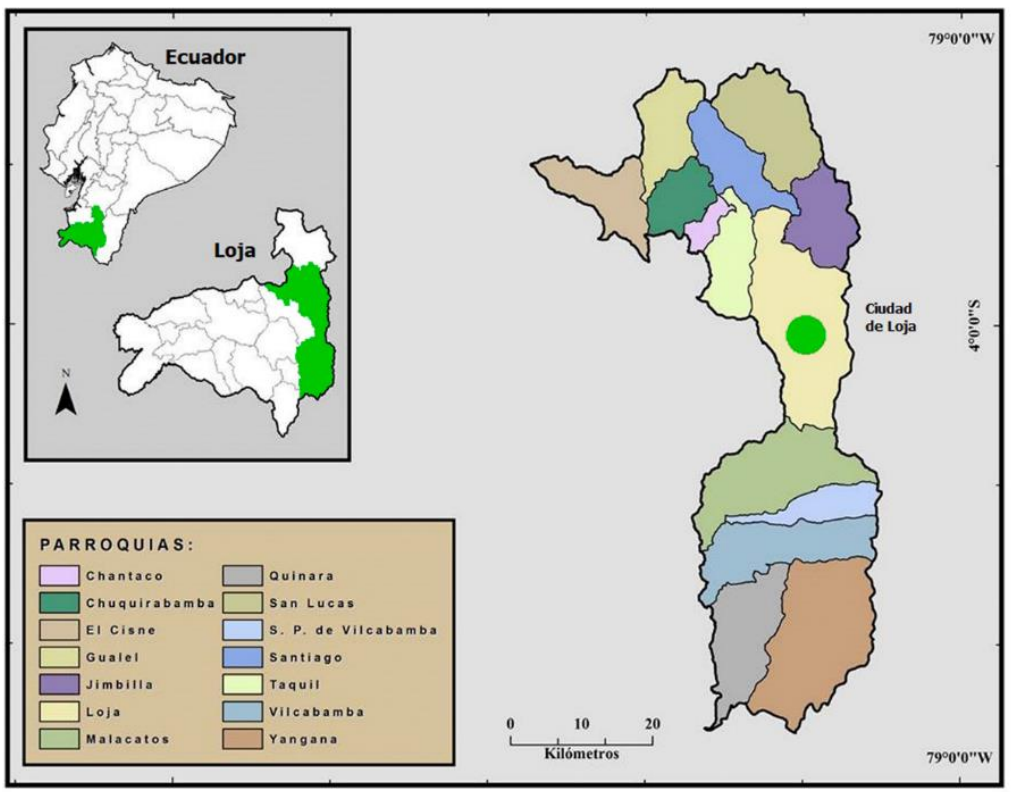

Figura 1. Mapa de ubicación de la ciudad de Loja

Fuente: Trabajo de campo

Elaborado por: Los autores

En función de estas características, se propone la creación del Festival Internacional de Artes Vivas de Loja, debido a que la ciudad dispone de una alta presencia de artistas en relación al número de habitantes que registra.

El propósito de este evento es convertir a la ciudad en un referente de las industrias culturales y creativas de las artes escénicas (siendo la música el campo de mayor interés), tanto a nivel nacional como internacional. El festival se organiza en función de una agenda de eventos dentro de los cuales se manifiestan de forma libre, diversas formas de expresión artística y lenguajes escénicos a partir de los cuales se impulsa la participación de artistas nacionales e internacionales (Festival Internacional de Artes Vivas Loja [FIAV], 2018), con lo cual se busca dar a conocer la importancia que el arte tiene para el Ecuador, al tiempo que transmite la cultura de este grupo humano hacia las audiencias visitantes del evento.

La creación de este festival ha permitido dar a conocer la diversidad artística y cultural de la ciudad, además de constituirse como una alternativa para la generación de empleos y el fomento del sector turístico de la región y el país. 


\section{Festival Internacional de Artes Vivas de Loja (FIAVL)}

Se define como un espacio de encuentro que impulsa la creación, disfrute y distribución de una amplia variedad de productos culturales, que permiten la revitalización de las manifestaciones culturales del pasado, todo ello con el firme propósito de contribuir en la cohesión social y en el fortalecimiento de las identidad lojana y ecuatoriana. Este festival, se propone contar con la plena participación de "todos los colectivos, estamentos, individuos, y personas con el fin de compartir bienes y servicios de contenido artístico y cultural tradicionales y vanguardistas, y para el ejercicio de sus derechos culturales" (Ley Orgánica para la Institucionalización del FIAVL 2019).

La organización del festival está a cargo del Ministerio de Cultura y Patrimonio (MCYP) como rector de la cultura en el país, quien se encarga de emitir las políticas, directrices y lineamientos necesarias para el desarrollo del FIAVL. Adicional a ello, éste complementa la organización del mismo, por medio de la coordinación de acciones conjuntas con la Casa de la Cultura Ecuatoriana "Benjamín Carrión" (CCE), el Gobierno Autónomo Descentralizado Municipal de Loja (GADM Loja), así como con las demás instituciones y actores del Sistema Nacional de Cultura que se adhieran, garantizando siempre la participación de actores y gestores culturales y/o sociales, así como instituciones públicas o privadas. La participación de estas instituciones podrá desarrollarse dentro de todas las fases que componen el festival. Adicional a ello, se dispone que la programación del evento sea de carácter anual ininterrumpido, permanente y obligatorio de ejecución durante el mes de noviembre dentro de la ciudad de Loja; con lo cual, a partir de la institucionalización del FIAVL el 14 de enero de 2019, se declara y reconoce a la ciudad de Loja como la "Capital Cultural y Musical del Ecuador en virtud de su raigambre académica y artística que ha contribuido a la construcción y fortalecimiento de la identidad cultural del país" (Ley Orgánica para la Institucionalización del FIAVL 2019). 


\section{Responsabilidades de las Instituaciones en el FIAVL}

Sedes logísticas, operativas y protocolarías

CCE

"Benjamín

Carrión"
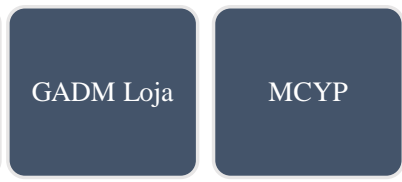

Generadores de los recursos

necesarios para la ejecución

dentro de la planificación anual

presupuestaria, esto no implica

aumento del gasto público.

estratégicas público-privadas

la ejecución, así como

la ejecución, así como
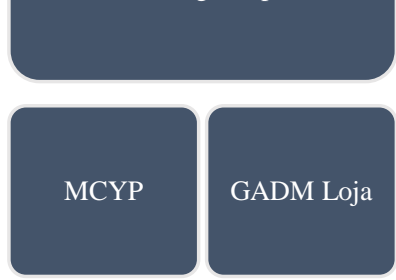

Figura 2. Responsabilidades por institución para la ejecución del FIAVL

Fuente: Ley Orgánica para la Institucionalización del FIAVL (2019)

Elaborado por: Los autores

La Figura 2 presenta la distribución de responsabilidades para la ejecución del festival en función de las instituciones participantes. Adicional a ello, se indica que el Instituto de Fomento de las Artes, Innovación y Creatividades (IFAIC) destinará un porcentaje de su presupuesto para contribuir en el financiamiento del fomento, la promoción y difusión de las actividades programadas para el FIAVL, los cuales serán de carácter concursable y no reembolsable.

\section{Loja y la Red de Ciudades Creativas de la UNESCO}

La ciudad de Loja desde los últimos cinco años ha iniciado la construcción del expediente de candidatura para la inserción de esta ciudad dentro de la iniciativa Red de Ciudades Creativas (RCC) desarrolla por la UNESCO, la cual agrupa 180 ciudades en 72 países del mundo. Estas ciudades se caracterizan por sus capacidades, habilidades y poblaciones con el firme objetivo de trabajar juntas para incentivar las industrias creativas. Este proyecto se inicia en 2004, donde se toma como eje central de trabajo el uso de la creatividad para el desarrollo urbano sostenible de los territorios (Unesco s.f.).

El interés que posee la ciudad por integrarse a esta red se encuentra asociado a sus designaciones nacionales, que se fundamentan en la larga línea de artistas nacionales e 
internacionales, estilos musicales y letras provenientes de esta latitud, así como, por el elevado número de personas dedicadas al estudio formal de la música y por la variedad de residentes que desde la informalidad diseminan la música convirtiéndola en una marca para esta ciudad. Adicional a ello, se busca estimular su capacidad creativa, social y económica, al generar espacios de encuentro e interculturalidad (Loja es música 2004).

En la actualidad dentro de la red se parecían 31 ciudades inscritas como parte de la categoría música, las cuales destacan por la diversidad urbana que poseen, así como las trasformaciones en sus políticas públicas que respaldan a la cultura como pilar de trabajo.

En estas ciudades resalta la existencia de una agenda cultural apoyada en la programación de festivales tanto gratuitos como pagados que están a disposición de los ciudadanos y que refuerzan la identidad y la diversidad cultural, al mismo tiempo que se convierten en puntos de atracción turística.

Tabla 1. Distribución de ciudades creativas de la música en la RCC

\begin{tabular}{|c|c|c|}
\hline Continente & País & Cantidad \\
\hline \multirow{6}{*}{ América } & Colombia & 2 \\
\hline & Estados Unidos & 2 \\
\hline & Brasil & 1 \\
\hline & Chile & 1 \\
\hline & Jamaica & 1 \\
\hline & México & 1 \\
\hline \multirow{8}{*}{ Europa } & Alemania & 2 \\
\hline & Italia & 2 \\
\hline & Portugal & 2 \\
\hline & Reino Unido & 2 \\
\hline & Bélgica & 1 \\
\hline & España & 1 \\
\hline & Polonia & 1 \\
\hline & Suecia & 1 \\
\hline \multirow{3}{*}{ África } & Cabo Verde & 1 \\
\hline & República del Congo & 1 \\
\hline & República Democrática del Congo & 1 \\
\hline \multirow{4}{*}{ Asia } & Corea del Sur & 2 \\
\hline & India & 2 \\
\hline & Japón & 1 \\
\hline & Kirguistán & 1 \\
\hline \multirow{2}{*}{ Oceanía } & Australia & 1 \\
\hline & Nueva Zelanda & 1 \\
\hline Total & & 31 \\
\hline
\end{tabular}

Fuente: Unesco (s.f.)

Elaborado por: Los autores 
En el caso de la ciudad de Loja, se dispone de una cartelera permanente de conciertos, eventos y festivales relacionados con este arte. En función de la base de datos de Loja es música $(2004)^{5}$, se conoce de la presencia de 645 artistas entre cantantes, instrumentistas, compositores, y cantautores, así como 15 grupos musicales que forman parte de la programación regular de los eventos. Además, se promueve una programación complementaria de otras tipologías de artes escénicas.

Cabe mencionar que, la ciudad dispone de infraestructuras para albergar todas estas manifestaciones. Uno de las más representativas es el Teatro Nacional Benjamín Carrión, el cual entro en funcionamiento durante la inauguración de la primera edición del FIAVL en 2016 (Ministerio de Turismo 2016).

\section{Base legal para la institucionalización del FIAVL}

El FIAVL fundamenta su accionar en el Art. 22 de la Constitución de la República del Ecuador (2008), mismo que manifiesta que todas "las personas tienen derecho a desarrollar su capacidad creativa, al ejercicio digno y sostenido de las actividades culturales y artísticas, y a beneficiarse de la protección de los derechos morales y patrimoniales que les correspondan por las producciones científicas, literarias o artísticas de su autoría”. (p.27) Así también, promueve lo manifestado en el Art. 23 a través de la creación de espacios públicos que permitan el intercambio cultural, la cohesión social y la promoción de la igualdad, así como el derecho a difundir todas las expresiones culturales existentes, para ello se determina que será competencia de los Gobiernos Autónomos Descentralizados Municipales la planificación, construcción y mantenimiento de dichos espacios públicos que contribuyen al desarrollo social, cultural y deportivo tal como indica el Art. 264 numerales 7 y 8 (Constitución de la República del Ecuador 2008).

También, se manifiesta por medio del Art. 377 la necesidad de incentivar "la libre creación artística y la producción, difusión, distribución y disfrute de bienes y servicios culturales; y salvaguardar la memoria social y el patrimonio cultural” (p.170). Este último, se promoverá

\footnotetext{
${ }^{5}$ Página oficial creada para la recopilación de información que contribuye al expediente de candidatura para "Loja, ciudad creativa de la música".
} 
tanto en lo tangible como intangible puesto es relevante para la memoria e identidad de las personas y colectivos.

Finalmente, Ley Orgánica para la Institucionalización del FIAVL (2019) determina que el festival "se proyecta como un importante punto de encuentro cultural en el mapa nacional, regional y mundial, que permitirá difundir el potencial cultural del país en sus diferentes aristas y facetas; así como fortalecer nuestra identidad y nuestras relaciones interculturales, por lo cual es necesario se proceda a su institucionalización legal”. (p.3)

\section{La cultura como eje de transformación de la economía}

Dentro del país, la Ley Orgánica de Cultura (2016) plantea entre sus fines como cuerpo legal el "reconocer e incentivar el aporte a la economía de las industrias culturales y creativas" (p.4), esta tendencia ha tomado fuerza en las últimas décadas, puntualmente se ha observado el aprovechamiento de los festivales como elementos para la reactivación económica (Devesa, Herrero, y Sanz 2009), puesto que éstos se están convirtiendo en una parte cada vez más importante de los destinos turísticos en muchas economías (Ferdinand y Williams 2013). Los festivales se catalogan por Holbrook y Hirschman (1982) como productos culturales singulares, debido a que tienen la capacidad de generar una experiencia específica para un público en particular (Andersson y Getz 2009), a partir de la articulación y vinculación de una diversidad de expresiones que se caracterizan por ser de consumo espontaneo, muy perecibles y heterogéneas (Poulsson y Kale 2004), además de provocar una serie de respuestas multisensoriales entre sus consumidores (Holbrook y Hirschman 1982).

La incorporación de este tipo de eventos se ha catalogado como una acción capaz de generar cambios favorables en destinos turísticos maduros, o convertirse en una alternativa que impulse el turismo en aquellos espacios que carecen de atractivos (Perles 2004, Perez 2016). Por la relevancia que estos productos han tomado, se ha iniciado el desarrollo de la economía naranja que se caracteriza por la comercialización de bienes y servicios que disponen de una capacidad innata para provocar la creatividad de las personas, convirtiéndolos en una auténtica fuente inagotable de recursos.

Por tal razón, la ciudad de Loja ha trabajado en la articulación del FIAVL como una las estrategias de desarrollo económico, al promover la actividad turística. Se observa que para 
2017 generó un ingreso económico por concepto de turismo de \$3’018,047.89 en la economía local (Universidad Técnica Particular de Loja 2017), valor que consiguió cubrir casi el total de lo que se invirtió para la ejecución del festival (Diario La Hora 2015). Es necesario mencionar que el festival registró para 2017 un presupuesto de USD 5 millones 828 mil dólares, valor que no fue empleado en su totalidad.

Tomando en consideración los ingresos generados por este evento, se aprecia como relevante el analizar la satisfacción del turista sobre los eventos más destacados del FIAVL, a través de un análisis de correspondencia simple y múltiple.

\section{Metodología}

\section{Área de estudio}

Se toma el II Festival Internacional de Artes Vivas de Loja (FIAVL), es decir, la edición 2017 del evento, la cual se celebró entre el 16 y 26 de noviembre de dicho año. Este festival contó con al menos 12 espectáculos internacionales procedentes de distintas partes de Europa y Latinoamérica, a partir de los cuales se puso en escena más de 10 géneros de artes escénicas.

En grandes rasgos, se cuantificó la participación de más de 5.060 artistas de los cuales 5.000 fueron artista locales, 42 nacionales y 18 internacionales, en alrededor de 30 obras en escena desarrolladas en 10 escenarios diferentes (Observatorio Turístico Región Sur de Ecuador UTPL y Ministerio de Turismo 2017). Los eventos programados fueron tanto de acceso libre como pagados.

\section{Recolección de datos}

Dentro del estudio se empleó un enfoque cuali-cuantitativo, integrado por la aplicación de procesos de revisión bibliográfica y análisis descriptivo. La técnica utilizada para recopilar información corresponde a la aplicación de una encuesta a los visitantes de los distintos eventos que conformaron la programación. Dentro del festival se registró la participación de aproximadamente 6.081 personas, dato que registra un incremento del $52 \%$ respecto a la primera edición del evento. 
El cálculo de la muestra determinó una recogida de 375 encuestas, que se integró por cuatro secciones: a) datos generales del evento y el turista, b) datos de la experiencia turística, c) organización y distribución del gasto y c) evaluación de Loja como destino turístico. El levantamiento de la información considero un muestro sistemático, hecho que permitió disponer de una mayor diversidad de opiniones.

\section{Análisis de datos}

Se inicia el análisis a través de la descripción de las características sociodemográficas de los visitantes encuestados y de su preferencia por los diferentes eventos del festival. Posteriormente, a través de un análisis de correspondencias se analiza la percepción sobre la calidad y grado de satisfacción de los eventos.

Tabla 2. Operacionalización de variables

\begin{tabular}{|c|c|c|c|c|}
\hline & Variables & Tipo & $\begin{array}{l}\text { Escala de } \\
\text { medición }\end{array}$ & Categoría \\
\hline \multirow{19}{*}{$\begin{array}{l}\text { Perfil } \\
\text { sociodemográfico } \\
\text { del visitante }\end{array}$} & \multirow{2}{*}{ Género } & \multirow{2}{*}{ Cualitativa } & \multirow{2}{*}{ Nominal } & 1 Hombre \\
\hline & & & & 2 Mujer \\
\hline & \multirow{5}{*}{ Edad } & \multirow{5}{*}{ Cuantitativa } & \multirow{5}{*}{ Ordinal } & $111-25$ \\
\hline & & & & $226-40$ \\
\hline & & & & $341-55$ \\
\hline & & & & $456-70$ \\
\hline & & & & $571-85$ \\
\hline & \multirow{5}{*}{ Nivel de instrucción } & \multirow{5}{*}{ Cualitativa } & \multirow{5}{*}{ Ordinal } & $0 \quad$ Ninguno \\
\hline & & & & 1 Primario \\
\hline & & & & 2 Secundario \\
\hline & & & & 3 Superior \\
\hline & & & & 4 Post Grado \\
\hline & \multirow{7}{*}{ Ingresos } & \multirow{7}{*}{ Cuantitativa } & \multirow{7}{*}{ Ordinal } & $1 \quad$ Menor de $\$ 500$ \\
\hline & & & & 2 De $\$ 500$ a $\$ 1000$ \\
\hline & & & & 3 De $\$ 1001$ a $\$ 1500$ \\
\hline & & & & 4 De $\$ 1501$ a $\$ 2000$ \\
\hline & & & & 5 De $\$ 2001$ a $\$ 2500$ \\
\hline & & & & 6 De $\$ 2501$ a $\$ 3000$ \\
\hline & & & & 7 De \$3001 en adelante \\
\hline
\end{tabular}


ISSN: 2602-8085

Vol. 3, N³.4, p. 191-211, septiembre, 2019

\begin{tabular}{|c|c|c|c|c|}
\hline & Variables & Tipo & $\begin{array}{l}\text { Escala de } \\
\text { medición }\end{array}$ & Categoría \\
\hline \multirow{17}{*}{$\begin{array}{l}\text { Percepción de los } \\
\text { eventos del FIAVL }\end{array}$} & \multirow{4}{*}{ Tipo de eventos } & \multirow{4}{*}{ Cualitativa } & \multirow{4}{*}{ Nominal } & 1 Teatro \\
\hline & & & & 2 Danza \\
\hline & & & & 3 Canto \\
\hline & & & & 4 Otro \\
\hline & \multirow{3}{*}{$\begin{array}{l}\text { ¿Los eventos en que } \\
\text { participó fueron? }\end{array}$} & \multirow{3}{*}{ Cualitativa } & \multirow{3}{*}{ Nominal } & 1 Pagados \\
\hline & & & & 2 Libres \\
\hline & & & & 3 Ambos \\
\hline & \multirow{5}{*}{$\begin{array}{l}\text { Satisfacción en relación } \\
\text { con el precio de los } \\
\text { eventos }\end{array}$} & \multirow{5}{*}{ Cualitativa } & \multirow{5}{*}{ Ordinal } & 1 Nada satisfecho \\
\hline & & & & 2 Poco satisfecho \\
\hline & & & & 3 Ni satisfecho ni insatisfecho \\
\hline & & & & 4 Satisfecho \\
\hline & & & & 5 Muy satisfecho \\
\hline & \multirow{5}{*}{$\begin{array}{l}\text { ¿Recomendaría el } \\
\text { FIAVL a otras } \\
\text { personas? }\end{array}$} & \multirow{5}{*}{ Cualitativa } & \multirow{5}{*}{ Ordinal } & 1 Para nada recomendable \\
\hline & & & & 2 Poco recomendable \\
\hline & & & & $\begin{array}{ll}\text { Ni recomendable ni no } \\
\text { recomendable }\end{array}$ \\
\hline & & & & 4 Recomendable \\
\hline & & & & 5 Totalmente recomendable \\
\hline
\end{tabular}

Fuente: Trabajo de campo

Elaborado por: Los autores

El análisis de correspondencias (AC) es una técnica de análisis multivariado que permite describir la relación existente entre dos o más variables cualitativas en un diagrama bidimensional, basado en la relación de dependencia e independencia de los atributos o categorías de las variables. La estrecha relación que existe entre las categorías de las variables se visualiza por su cercanía en el diagrama; de ahí se deriva el nombre de mapas perceptuales que éstos reciben.

\section{Resultados}

La encuesta estuvo dirigida a 375 visitantes, el 97\% de ellos fueron nacionales y el 3\% extranjeros provenientes de Estados Unidos, Colombia y Brasil (Figura 3); los eventos con mayor acogida fueron los relacionados a teatro con una aceptación del 66\%, mientras que danza y canto se encuentran seguidos por el $15 \%$ y $9 \%$ respectivamente, y en cuanto al género se reporta que el $54 \%$ fueron mujeres (Figura 4). 


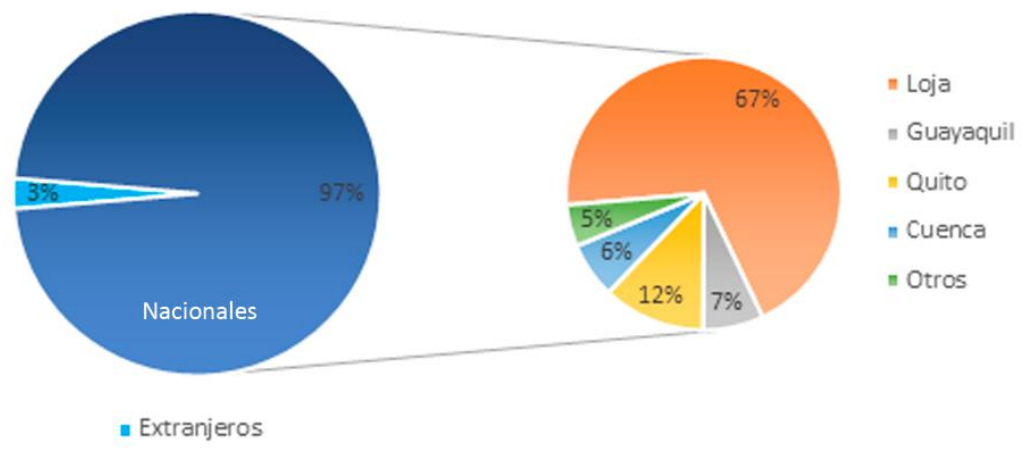

Figura 3. Lugar de procedencia del visitante Fuente: Trabajo de campo

Elaborado por: Los autores

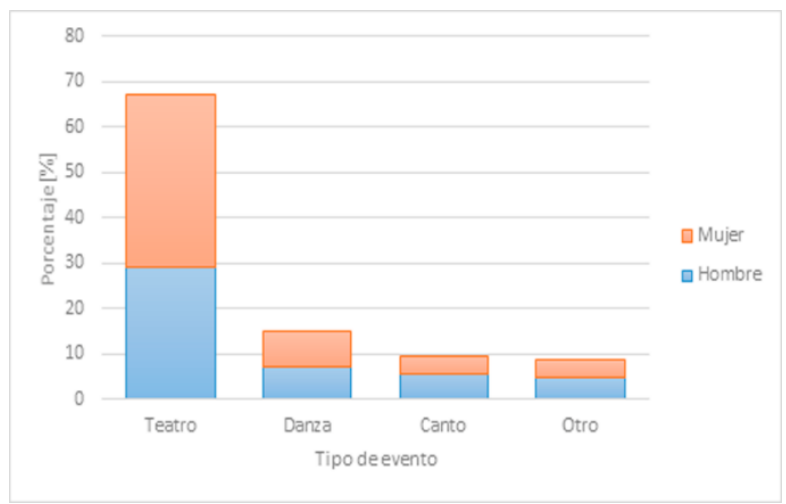

Figura 4. Género por tipo de evento

Fuente: Trabajo de campo

Elaborado por: Los autores

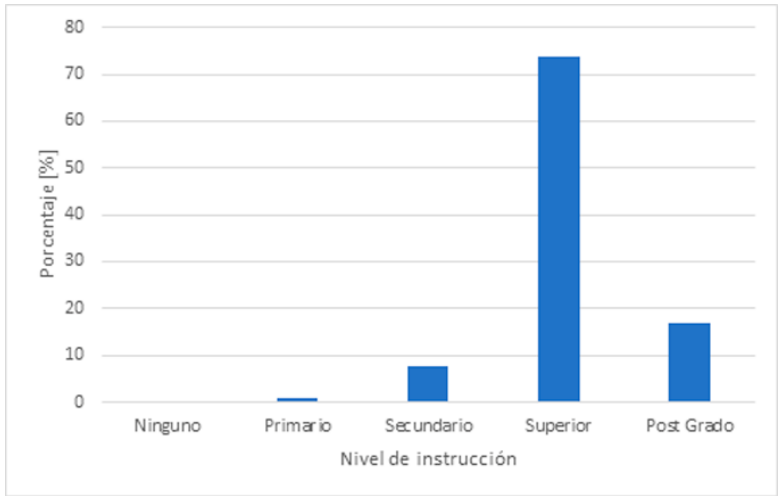

Figura 5. Nivel de instrucción

Fuente: Trabajo de campo

Elaborado por: Los autores 
En la Figura 5, se identifica que el 90\% de los visitantes, al menos han alcanzado un nivel de instrucción superior, resultado que provoca un cambio en el perfil del visitante, debido a la atracción de personas que disponen de un mayor gasto y al mismo tiempo un mayor interés sobre los aspectos culturales.

Seguido, la Figura 6 revela una tendencia decreciente en el porcentaje de visitantes que poseen mayores ingresos. Tan sólo un 13\% de visitantes perciben ingresos mensuales superiores a 2.000 dólares; en el caso opuesto, el $27 \%$ percibe ingresos menores a 500 dólares. Cabe recalcar que este último grupo se encuentra representado (21\%) por personas cuya edad máxima es 25 años.

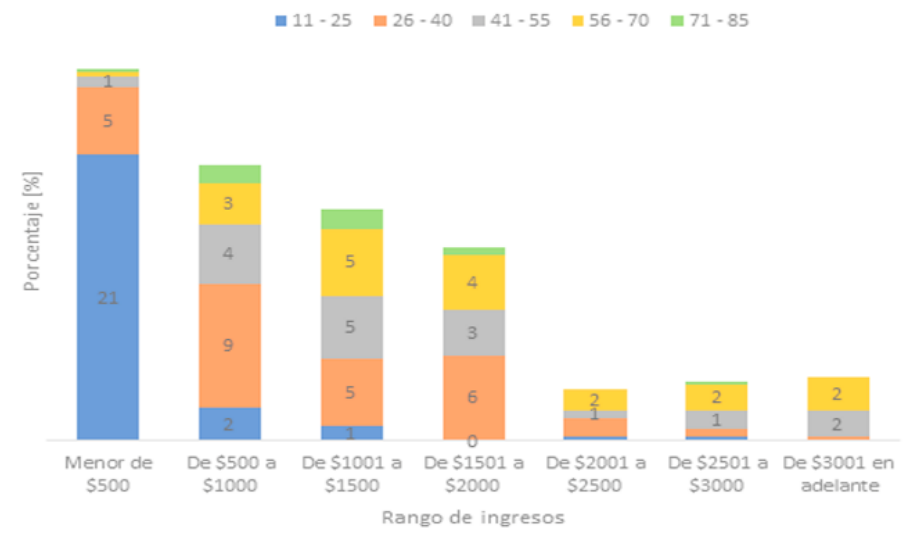

Figura 6. Rango de ingresos por edad

Fuente: Trabajo de campo

Elaborado por: Los autores

En la Figura 7, se visualiza la relación que existe entre los eventos desarrollados en el FIAVL, en la cual se aprecia que el pagar por asistir a un evento no está directamente relacionado a una tipología de evento en especial, es decir se pueden encontrar eventos de teatro, música y canto que son tanto libres como pagados; a pesar de ello, existe una gran acogida por los eventos pagados (Figura 8). 


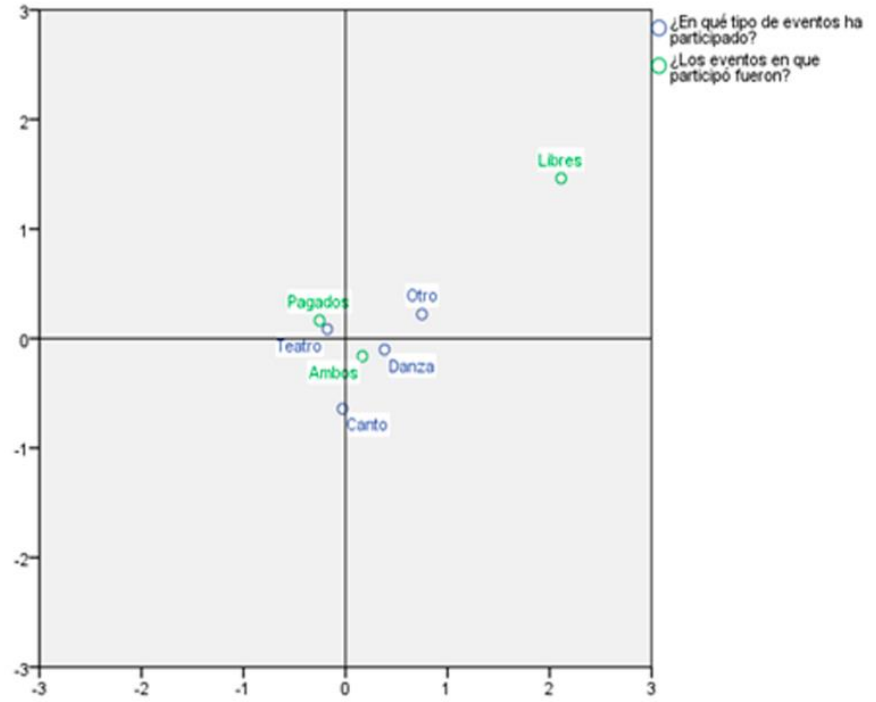

Figura 7. Mapa perceptual de la relación entre eventos

Fuente: Trabajo de campo

Elaborado por: Los autores

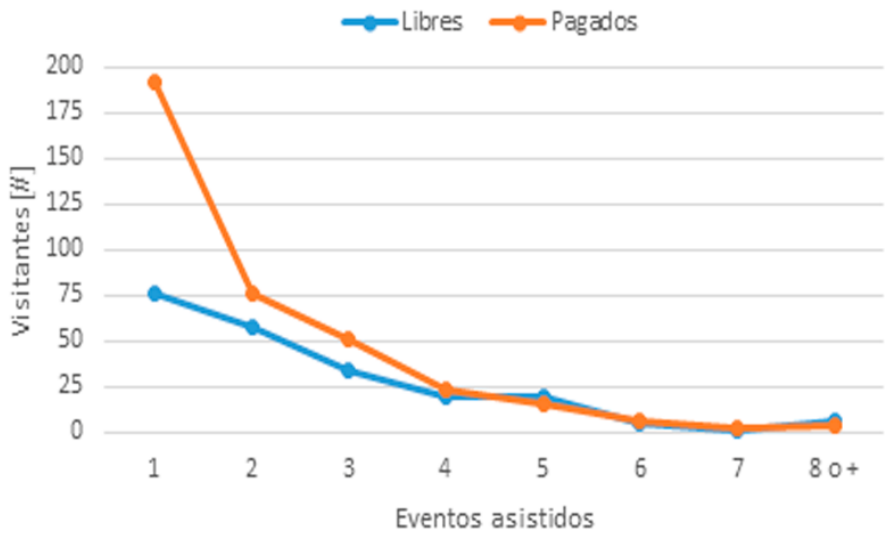

Figura 8. Distribución de número de visitantes a eventos asistidos

Fuente: Trabajo de campo

Elaborado por: Los autores

En cuanto al grado de satisfacción, los visitantes de manera general se encuentran satisfechos o muy satisfechos por los eventos a los que han acudido, tomando en consideración el precio del mismo (Figura 9). 


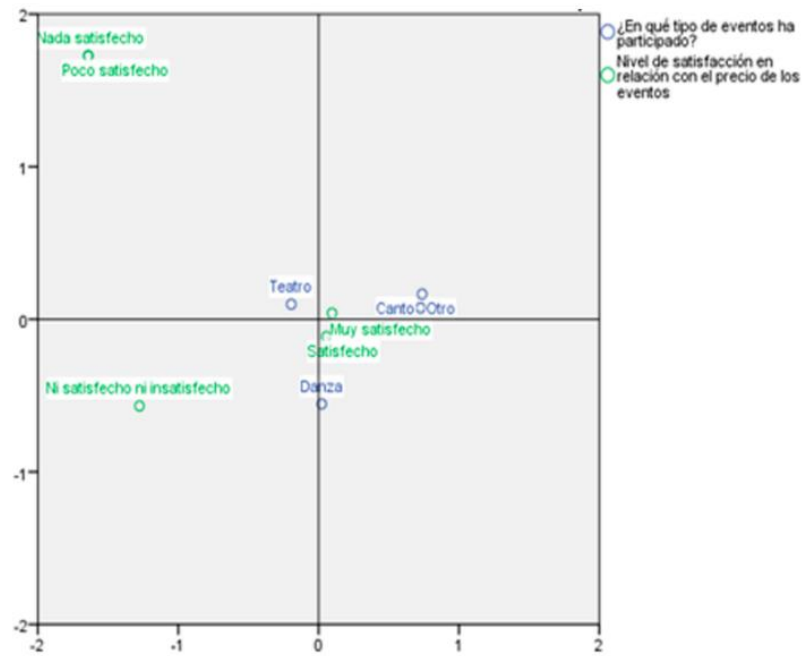

Figura 9. Mapa perceptual de la relación de los tipos de evento y su satisfacción Fuente: Trabajo de campo

Elaborado por: Los autores

Así mismo, en base a la experiencia vivida en el FIAVL se observa que los visitantes recomendarían a otras personas participar en él, donde los eventos que serían totalmente recomendables se encuentran más relacionados al canto y el teatro (Figura 10).

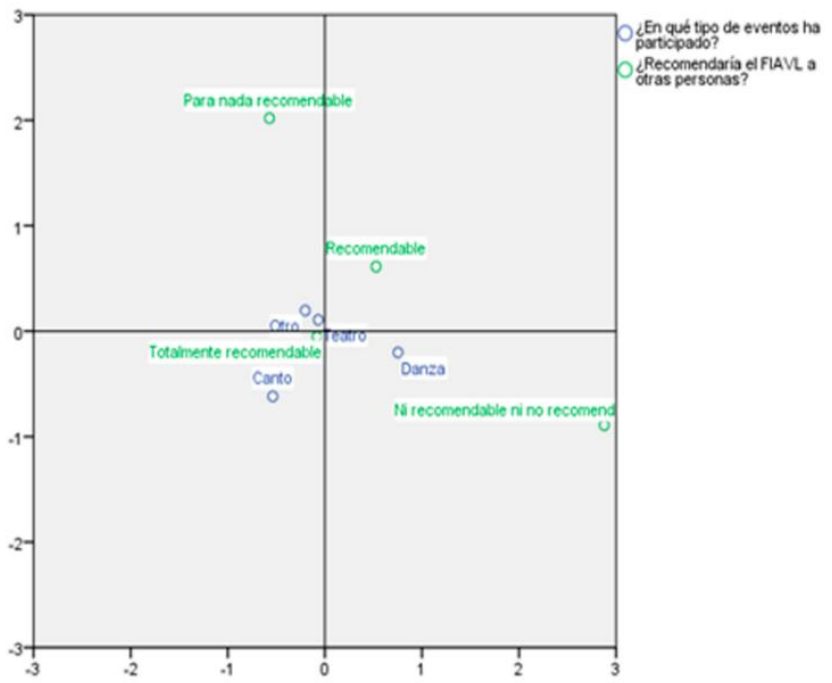

Figura 10. Mapa perceptual de la relación de los tipos de eventos y su recomendación Fuente: Trabajo de campo

Elaborado por: Los autores 
A continuación, se detalla el análisis realizado entre cinco variables que pretenden determinar la relación existente entre la satisfacción del visitante y sus características socioeconómicas, reduciendo su descripción a un espacio de dos dimensiones.

Tabla 3. Resumen del modelo del análisis de correspondencias

\begin{tabular}{|c|c|c|c|}
\hline \multirow[t]{2}{*}{ Dimensión } & \multirow[t]{2}{*}{$\begin{array}{c}\text { Alfa de } \\
\text { Cronbach }\end{array}$} & \multicolumn{2}{|c|}{ Varianza contabilizada para } \\
\hline & & $\begin{array}{c}\text { Total } \\
\text { (autovalor) }\end{array}$ & Inercia \\
\hline 1 & ,638 & 2,041 & ,408 \\
\hline 2 & ,460 & 1,583 & ,317 \\
\hline Total & & 3,624 &, 725 \\
\hline Media &, $560 *$ & 1,812 & ,362 \\
\hline
\end{tabular}

* La media de alfa de Cronbach se basa en la media de autovalor.

Fuente: Trabajo de campo

Elaborado por: Los autores

Se observa que hay un 72,5\% de la variabilidad de los datos que está siendo explicada por las dimensiones incluidas en este modelo. La primera dimensión aporta el 56\% $(0,408 / 0,725)$ de la variabilidad explicada, mientras que la segunda dimensión explica el $44 \%$ $(0,317 / 0,725)$. En cuanto a la fiabilidad de la escala de medida de las variables empleadas, el coeficiente Alfa de Cronbach presenta un valor promedio 0,638, el cual como máximo puede llegar a 1.

La primera dimensión se encuentra representada en mayor medida por la edad y el ingreso económico, mientras que la segunda categoría está representada por el nivel de instrucción, la edad y el tipo de evento.

En la Figura 11, se observan las relaciones entre las características socioeconómicas de los visitantes y los eventos a los que han acudido. Se observa que los visitantes cuyo nivel de instrucción es de postgrado, se encuentran en edades comprendidas entre los 41-70 años cuyos ingresos son superiores a los 1.000 dólares y prefieren en cierta medida los eventos de danza y teatro; mientras que, para quienes tienen una instrucción secundaria su edad presenta mayor variabilidad, sus ingresos son menores a 500 dólares y su nivel de satisfacción es intermedio tomando en cuenta que prefieren los eventos de danza y canto. 


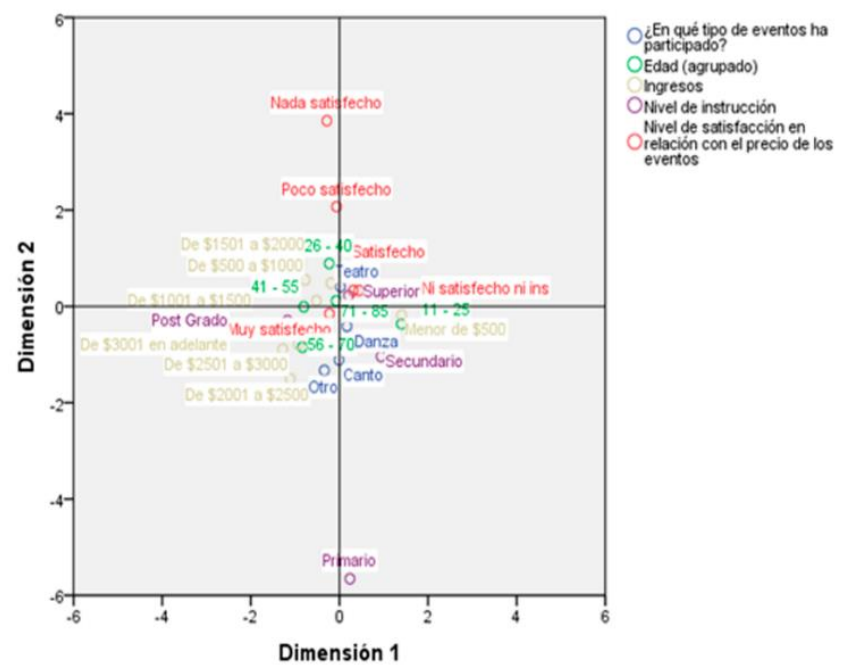

Figura 11. Mapa perceptual de la relación de los tipos de eventos, edad, ingresos, nivel de instrucción y satisfacción del evento/precio

Fuente: Trabajo de campo

Elaborado por: Los autores

\section{Discusión}

Durante las dos primeras ediciones del festival, éste se ha dado a conocer como un referente nacional para la expresión de todo tipo de artes escénicas, las cuales de acuerdo al modelo del Instituto de Estadísticas de la Unesco se establecen como ámbitos culturales fundamentales para las industrias culturales y creativas (Unesco 2007).

De esta manera, se aprecia que el FIAVL centra la cartelera de eventos en una amplia variedad de artes escénicas, pero es el teatro la tipología predominante dentro de la programación, dejando al canto en un segundo plano. Adicional a ello, un gran porcentaje de eventos de carácter pagado corresponden a teatro, siendo el canto una propuesta predominante dentro de los eventos libres.

Con respecto a la afluencia de los visitantes, en función de la nacionalidad se aprecia que por cada 100 nacionales 2 son extranjeros; en tanto que, por cada 10 residentes 5 son visitantes. La edad de los asistentes se encuentra en el rango de 11 a 83 años, siendo sobre el 50\% personas menores a 40 años, que prefieren eventos relacionados al canto; mientras que el 
porcentaje restante (mayor a 40 años) muestra predilección por asistir a eventos de danza y teatro. Así también, se establece que la mayoría más del 90\% han alcanzado estudios superiores y de posgrado, factor que refuerza la teoría de (Shackley 2006) sobre el aumento de la especialización del perfil del turista hacia destinos culturales.

Además, la percepción de calidad del festival en un $94 \%$ de los visitantes se encuentra entre buena y muy buena; y en un porcentaje similar $(98,4 \%)$ los visitantes recomendarían el evento a otras personas.

De forma general, se puede mencionar que solo el 14\% de los participantes presentaron problemas dentro del FIAVL, a pesar de ello, más del $90 \%$ de todos los visitantes están satisfechos, recomendarían y tienen la predisposición de asistir a la edición del 2018.

Finalmente, se puede indicar que existe aceptación hacia los eventos de canto, cuya percepción de calidad es muy buena, motivo por el cual se está contribuyendo a la declaratoria; sin embargo, esta tipología no es el evento focal por el que acuden los visitantes, siendo necesario mejorar el posicionamiento de esta tipología del arte, con el propósito de evidenciar un aporte de mayor significancia para el expediente en construcción.

\section{Conclusiones.}

- Los hallazgos del estudio evidencian que la localización de la ciudad no es una limitante para su visita al registrar un aumento de los visitantes del $52 \%$ en comparación a la primera edición.

- Entre las limitaciones identificadas se aprecia que, por la gran variedad de espacios para la generación de los eventos programados, se dificulta una cobertura global de todos los escenarios hecho que reduce la diversidad de opiniones vertidas por los visitantes.

- Se debe mencionar que, por inmensa cantidad de eventos de carácter informal programados en las calles de la ciudad, no ha sido posible cuantificar exactamente el impacto socio-económico que causa el festival.

- Adicional a ello, la fuerte participación de la ciudadanía dentro de las actividades programadas, ha provocado que la mayoría de la información recabada provenga de 
residentes, siendo necesario fortalecer los filtros de encuestas dentro del proceso de levantamiento de información.

- La ciudad debe trabajar en estrategias de gestión que permitan la incorporación de los artistas registrados dentro de la base de datos de la candidatura para ciudad creativa, acción que dará mayor sustento a lo propuesto dentro del expediente desarrollado para la Unesco.

\section{Referencias bibliográficas.}

Andersson, T. D., y D. Getz. 2009. "Tourism as a mixed industry: differences between private, public and not for profit festivals." Tourism Management no. 30 (6):847-856.

Constitución de la República del Ecuador. 2008. Registro Oficial 449 de 20 de octubre de 2008. Montecristi: Asamblea Constituyente.

Devesa, M., L. C. Herrero, y J. A. Sanz. 2009. "Análisis económico de la demanda de un festival cultural." Estudios de Economía Aplicada no. 27 (1):137-158.

Diario La Hora. 2017. Turismo mejoró en el FIAV de Loja. Diario La Hora, https://lahora.com.ec/loja/noticia/1102123524/turismo-mejoro-en-el-fiavl-de-loja [consultado el 17 de septiembre, 2019].

Ferdinand, N., y N. L. Williams. 2013. "International festivals as experience production systems." Tourism Management no. 34:202-210. doi: 10.1016/j.tourman.2012.05.001.

Holbrook, M. B., y E. C. Hirschman. 1982. "The experiential aspects of consumption: consumer fantasies, feelings, and fun." Journal of Consumer Research no. 9 (2):132140.

Ley Orgánica para la Institucionalización del FIAVL. 2019. Registro Oficial No.405 lunes 14 de enero de 2019.

Loja es música. 2004. Loja, camino a ciudad creativa de la música, https://lojaesmusica.com/about [consultado el 17 de septiembre, 2019].

Ministerio de Turismo. 2016. Se inauguró oficialmente el Primer Festival Internacional de Artes Vivas de Loja, https://www.turismo.gob.ec/se-inauguro-oficialmente-el- 
primer-festival-internacional-de-artes-vivas-de-loja/ [consultado el 17 de septiembre, 2019].

Observatorio Turístico Región Sur de Ecuador - UTPL, y Ministerio de Turismo. 2017. Reporte estadístico II Festival de Artes Vivas Loja 2017. Loja: UTPL.

Oviedo, J. 2012. A orillas del Zamora. Loja: Diario La Hora.

Pérez, L. 2016. Impactos turístico-económicos y socio-culturales de los festivales musicales en la comunidad valenciana, Universidad de Alicante, Alicante.

Perles, J. F. 2004. Turismo, Ventaja Competitiva y Desarrollo Local, Departamento de Análisis Económico Aplicado, Universidad de Alicante, Alicante.

Poulsson, S. H., y S. H. Kale. 2004. "The experience economy and commercial experiences." Marketing Review no. 4 (3):267-277.

Shackley, M. 2006. "Visitor management at World Heritage Sites." In Managing World Heritage Sites, editado por A. Leask y A. Fyall, 83-94. London: ButterworthHeinemann (Elsevier).

Unesco. 2007. Statistics on cultural industries: Framework for the elaboration of national data capacity building projects. Bangkok: Office of the UNESCO Regional Advisor for Culture in Asia and the Pacific: UNESCO Institute for Statistics (UIS).

Unesco. s.f. Red de Ciudades Creativas, https://es.unesco.org/creativecities/content/ciudades-creativas [consultado el 17 de septiembre, 2019].

Universidad Técnica Particular de Loja. 2017. Presentación de estadísticas turísticas del II FIAVL 2017. UTPL, https://noticias.utpl.edu.ec/sala-prensa/?q=node/60 [consultado el 17 de septiembre, 2019].

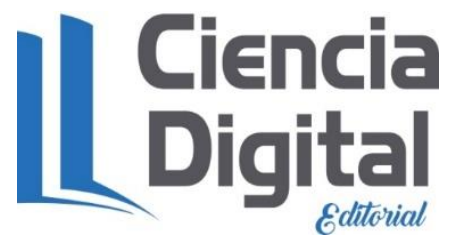


PARA CITAR EL ARTÍCULO INDEXADO.

Flores Mancheno, A. C., Maldonado Erazo, C., Tierra Tierra, N., \& Gallegos Murillo, P. de L. (2019). Satisfacción del turista en los eventos del FIAV para la declaratoria de "Loja, ciudad creativa de la música". Ciencia Digital, 3(3.4.), 191-211. https://doi.org/10.33262/cienciadigital.v3i3.4.846

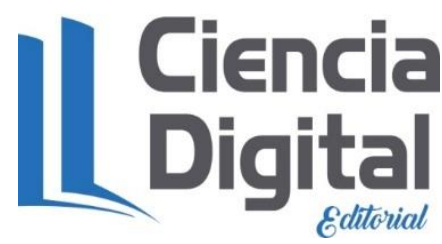

El artículo que se publica es de exclusiva responsabilidad de los autores y no necesariamente reflejan el pensamiento de la Revista Ciencia Digital.

El artículo queda en propiedad de la revista y, por tanto, su publicación parcial y/o total en otro medio tiene que ser autorizado por el director de la Revista Ciencia Digital.
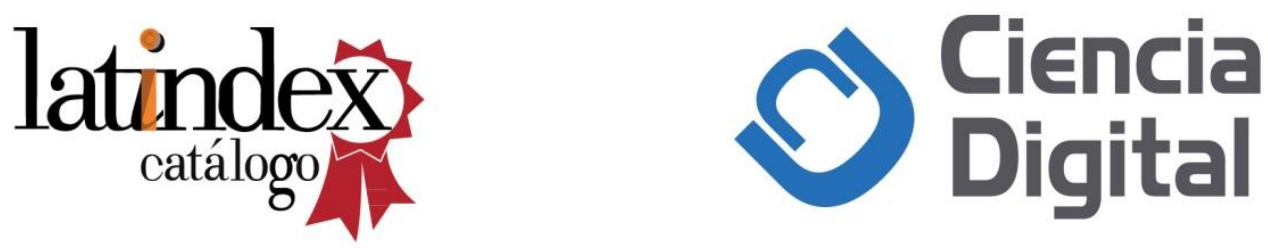